\title{
LA NACIÓN, LA DIÁSPORA Y LO SOCIAL DISPERSO
}

\section{The Nation, the diaspora and the social spread}

\author{
Ignacio Irazuzta* \\ * Tecnologico de Monterrey (México) \\ ignacio.irazuzta@gmail.com
}

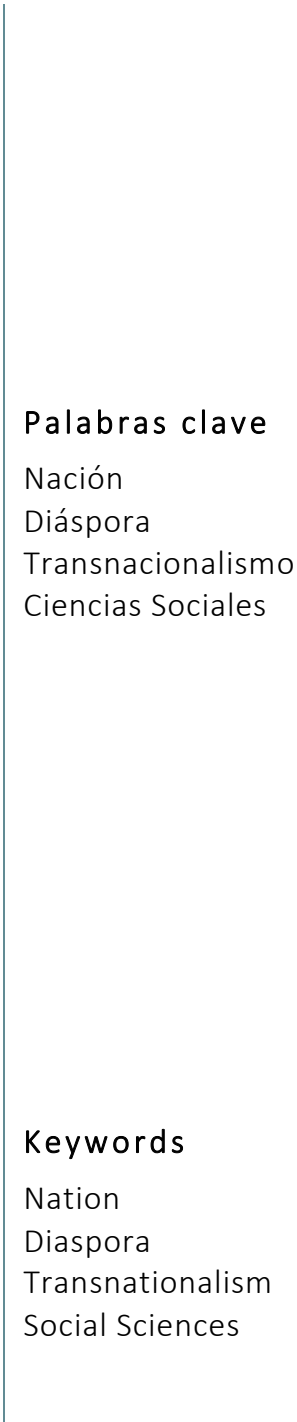

\begin{abstract}
Resumen
El artículo pone en relación el tema del Estado-nación y el nacionalismo en la obra de Alfonso Pérez-Agote con los debates acerca de las diásporas y el transnacionalismo que despuntan en las ciencias sociales de los años noventa. En el primer apartado, partiendo de la distinción entre Estado y nación como estrategia analítica inicial en dicha obra, se señalan los principales puntos que hacen al proceso de construcción de la realidad social en la modernidad para indicar la conformación de un orden social y subjetivo acotado a la delimitación territorial del Estado-nación. En el segundo, se introducen los principales términos de la problematización sobre las diásporas y el transnacionalismo en las ciencias sociales, subrayando las tendencias hacia la desterritorialización del vínculo social y la emergencia sobreexpuesta de las subjetividades que dichas tendencias provocan. Se sugiere así el paso de una sociedad acotada a la medida territorial del Estado-nación a un orden social disperso en el que la nación excede al Estado y el sujeto se descentra respecto a las instituciones que gestionan la legitimidad en la era del Estado-nación.
\end{abstract}

\begin{abstract}
This article bridges the topics of the Nation-State and nationalism in the works of Alfonso Pérez-Agote to the debates on diasporas and trans-nationalism, which prominently emerged in Social Sciences in the decade of the 1990's. In its first part, by exploring the distinction between Nation and State, we engage in an initial analytical strategy of this author's work. The main focus is on the construction of a social reality in Modernity that nurtured the construction of a system that is both social by definition and subjective by nature within a delimited and localized territory known as the Nation-State. In the second part, the major terms of the specific situational problem are introduced, focusing mainly on diasporas and transnationalism in the realm of social sciences. There we discuss the prevalence of tendencies towards de-territorialization of the social connection and the over-exposed emergence of subjectivities that these tendencies instigate. Thus we address the flow from a delimited and localized society conformed to the territoriality of the Nation-State to a dispersed social order in which the Nation exceeds and surpasses the State itself and leaves the Subject as a decentralized figure in regard to the institutions that negotiate legitimacy in the era on the Nation-State.
\end{abstract}

Irazuzta, I., 2015, "La nación, la diáspora y lo social disperso", en Papeles de/ CEIC, vol. 2015/3, no 140, CEIC (Centro de Estudios sobre la Identidad Colectiva), Universidad del País Vasco, http://dx.doi.org/10.1387/pceic.15183

Recibido: 11/2015; Aceptado: 11/2015 


\section{INTRODUCCIÓN}

Dedicar un artículo a algún tema o aspecto contenido en la obra de quien ha sido profesor de quien esto escribe enfrenta varios riesgos. Uno de ellos puede ser no haber aprendido la lección y reincidir aquí en malas interpretaciones sobre antiguas enseñanzas. Otro quizá sea sobredimensionar alguno de estos temas o aspectos dejándose llevar por la memoria de aquellas lecciones. Enfrento aqui ambos riesgos procurando desentrañar las marcas de aquel magisterio en uno de los temas que me he dedicado a estudiar durante mi desarrollo académico posterior. Entre aquellas lecciones y estos nuevos temas es donde, entiendo, radica la huella de la enseñanza y, ajena ya a la voluntad pretérita del profesor, podría resumirla en los siguientes términos: de Alfonso he hecho mía una Sociología que se esfuerza por vincular el sentido fluido de la experiencia social con los mecanismos sólidos que lo producen y reproducen; una premisa fuerte que es sensible al sentido de la relación social en la dedicación y la instrumentalización empírica para la producción de conocimiento.

El texto que sigue aborda uno de los temas que en su trayectoria de investigación Pérez-Agote ha sido trabajado con esa fortaleza y sensibilidad: el de la nación y los procesos de construcción de la realidad social que la producen y reproducen. Y lo pone posteriormente en relación con la cuestión de las diásporas como fenómenos de descentramiento y dispersión del sentido y de lo social respecto a la trama exclusivista del Estado-nación. La puesta en común de ambos temas permite plantear una serie de cuestiones a la luz de los principales cambios sociales contemporáneos, asuntos que están presentes como preocupaciones teóricas desde los primeros textos de Pérez-Agote: ¿qué desafíos lógicoanalíticos introduce una noción que evoca la dispersión - la de diásporacon respecto a otra - la de nación - que pareciera aludir a la concentración? ¿cómo abordan las ciencias sociales la emergencia de las diásporas en los tiempos transnacionales? ¿cómo podemos pensar lo social en estos tiempos?

Para el tratamiento de estas cuestiones el artículo presenta primero un apartado que recoge los principales planteamientos sobre la idea de nación en los trabajos de Pérez-Agote. Seguidamente, aborda la cuestión de las diásporas, su emergencia y tratamiento como categoría en las ciencias sociales y su relación con los procesos de transnacionalización de la vida social. Hacia el final, el texto profundiza en los interrogantes que 
plantea la puesta en común de ambos apartados. "Apartados" y "puesta en común" se revelan aquí como algo más que muletillas cuyo significado refiere a las estructuras de un texto académico; seguramente traducen el devenir de sentido de un relación también académica, aquella que hace a la de un profesor con su alumno ahora ya profesor.

\section{LA NACIÓN Y LO SOCIAL ACOTADO COMO SOCIEDAD}

La preocupación teórica por la nación es central en la obra de Pérez-Agote. Y quizá lo sea por el apremio de atender con explicaciones alternativas a las socialmente dispuestas en una realidad conflictiva, la del País Vasco, que presenta dos definiciones contrapuestas sobre el nosotros nacional: una fractura expuesta de sociedad que es tan apetente para el trabajo sociológico como especialmente didáctica para los aprendices del oficio. En este marco, su sociología del nacionalismo es una lógica explicativa de lo social que parte de los trazos más gruesos de la historia sin dejar nunca de "escuchar el enigma atentamente antes de intentar descifrarlo" (PérezAgote, 2008: 6). Su Sociología hace así del sentido encubierto de la relación entre Estado y nación una distinción analítica fundamental (TiryakianNevitte, en Pérez-Agote, 1989a), un punto de partida estratégico puesto que de él se derivan cinco supuestos esenciales que me interesa aquí destacar:

1. En primer lugar, cabría decir que es esta distinción inicial - la de Estado y nación- lo que le permite a Pérez-Agote ver y analizar el proceso de construcción de un orden social a la medida del Estado que lo produce. Es éste el que "corta y divide la realidad social indeterminada y, en cierto modo, difusa, en realidades sociales totales objetivadas" (Pérez-Agote, 1989b: 18). Recorte y división que es la delimitación territorial de la realidad social, espacio objetivo que, a través de su legitimación, se torna subjetivamente significativo como comunidad para los individuos que lo habitan'. Hay por lo tanto en este recorte una concepción

\footnotetext{
${ }^{1}$ Interesante y por tanto recomendable es el análisis que realiza nuestro autor en cuestión de la definición de Estado de Weber, especialmente en lo referido al carácter ambiguo de la noción de comunidad con la que empieza dicha definición. Ésta encierra tanto un sentido subjetivo que se desprende de la propia definición de weberiana de comunidad ("Ilamamos comunidad a una relación social cuando y en la medida en que la actitud en la relación social -en el caso particular, por término medio o en el tipo puro- se inspira en el sentimiento subjetivo (afectivo o tradicional) de los partícipes de constituir un todo"), como una connotación objetiva en la forma imprecisa de delimitación y corte de la realidad social (véase Pérez-Agote, 1989a: 183-185).
} 
instituyente de lo político, puesto que es lo que genera en un momento histórico el carácter total de lo social para devenir posteriormente como una realidad diferenciada de esa misma totalidad social que construye: "el momento político genera la sociedad, entendida como totalidad" (Pérez-Agote, 2012: 284). Esta prioridad lógica e histórica de lo político sobre lo social es lo que le permite a Pérez-Agote afirmar con rotundidad que la nación, ese "invento histórico occidental", es hija del Estado $^{2}$. y no al revés. Como orden lógico, y como tendencia históricamente dominante en Occidente, "la nación no tiene sentido si no es en referencia a un Estado" (Pérez-Agote, 1994: 25).

2. Es también esta distinción analítica entre Estado y nación lo que le posibilita al sociólogo evaluar la eficacia social de la construcción de la segunda por el primero, la capacidad de la nación para constituirse como una evidencia social, tener "éxito", en sentido weberiano. Cuando ello no sucede, cuando ese éxito no se alcanza, habrá que entender entonces que, de forma especular al proyecto central de nacionalización del Estado, se produce un "nacionalismo periférico" con respecto al central: "el fracaso del nacionalismo produce nacionalismo" (Pérez-Agote, 1994: 40). Una socialización defectuosa de la idea de nación puede producir en consecuencia la politización de determinados grupos étnicos preexistentes que reclamarán un Estado a la medida de su definición de la realidad. El nacionalismo, "movimiento tendente a la construcción de un Estado" (Ibid.: 24) posee asi un consecuente carácter teleológico; es un proyecto de futuro. De la consideración entonces de la eficacia social de la idea nación se derivan dos supuestos acerca del nacionalismo: un primero, que supone una idea de nación que emana del Estado y que produce una comunidad a la medida de ese Estado y su territorio; un segundo, que supone que "la idea de Nación es la idea de comunidad lanzada contra el Estado-Nación ya establecido, comunidad que dice estar amenazada en su existencia por ese Estado y que de forma más o menos expresa aspira a constituir el propio" (Pérez-Agote, 1989a: 179).

3. Lo que vincula a Estado y nación, lo que hace al guión que une y separa en la expresión Estado-nación, es del orden de lo simbólico subjetivo y se sintetiza en la noción de legitimidad. A los ojos de los individuos que habitan dicho Estado, la arbitrariedad que los constituye como grupo, la

\footnotetext{
${ }^{2}$ Aunque ese invento se realiza apoyado sobre elementos culturales preexistentes que, no obstante, no son todavía políticamente vinculantes ni "influyen en el pensamiento ni en los actos humanos en forma profunda y mensurable" (Pérez-Agote, 1989a: 179).
} 
violencia que funda el Estado, queda conjurada como Historia nacional que invierte los términos de la prioridad lógica del Estado sobre la nación: es el grupo, la nación, quien produce el Estado. Se trata de la producción de una legitimidad democrática que es arbitraria en cuanto a los rasgos que definen la existencia del grupo, pero no lo es en el sentido de que está determinada por procesos y condiciones históricos que producen y reproducen la "conciencia nacional". La producen a través de un mito fundacional; la reproducen a través de rituales civicos de desdiferenciación social (Pérez-Agote, 1994: 37-38; Irazuzta, 2001). Esta forma de producción simbólica del grupo, esta totalización cultural, tiene su otra cara, la racional, en la producción de un individuo (individuo-ciudadano) que es sujeto de derechos inalienables y que establece su relación con el Estado en términos impersonales (PérezAgote, 1989a: 180-182), arropada en la legitimidad legal-racional del dominio.

4. La separación analítica entre Estado y nación implica que lo social preexiste y excede a la sociedad. Preexiste puesto que, si el Estado reproduce lo social como una sociedad a su medida en tanto que "la sociedad es hija del Estado", ha de ser porque se ha impuesto sobre formas pretéritas de vinculación social. Pérez-Agote resalta esta cuestión exponiendo los argumentos de Nisbet cuando critica las posiciones del liberalismo conservador que oponen individuo a Estado. No es ello así porque, como se ha visto, la historia del individuo occidental moderno es la historia de la construcción del Estado igualmente moderno y territorial. Lo que está en juego entonces, lo que combate ese Estado, son las asociaciones tradicionales, como la familia, el gremio, el municipio, la parroquia..., en general, ese campanilismo frente al cual el individuo moderno va adquiriendo autonomía y aquellas viejas formas de vinculación social se van haciendo más débiles a medida que Estado e individuo-ciudadano se fortalecen (Pérez-Agote, 2012: 285). Y, en tanto que es esto un proceso histórico, podría decirse asimismo que lo social excede a la sociedad porque, como el mismo Pérez-Agote lo observa reproduciendo un planteamiento de Touraine (Cf. Touraine, 2005), si en nuestros días asistimos a una crisis de la sociedad, de una sociedad "regida por un centro" (Pérez-Agote, 2012: 285), ha de ser porque lo social se manifiesta de una forma descentrada, disgregada, dispersa..., desterritorializada, incluso. Una de las causas de este descentramiento es para Pérez-Agote la "mundialización", un proceso que implica la 
aparición de nuevas lógicas, lógicas englobantes, múltiples y complejas; nuevos centros, a veces desterritorializados, que producen $y$ reproducen nuevas subjetividades (Pérez-Agote, 1994: 41-42). Lo social se dispersa; también el nacionalismo, que parece reproducirse en formas que lo trascienden.

5. Finalmente, una preocupación también constante y temprana en la obra de Alfonso: la preocupación por la Sociología, que es tan deudora como responsable del proceso de construcción social descrito y de su devenir crítico. Algo más que una insinuación de "nacionalismo metodológico" hay en su apreciación al respecto puesto que, entre otras implicancias críticas, se observa una crisis del nativismo en la interpretación de la modernidad y el cambio social y una duda respecto a si, en esta disciplina, se puede seguir pensando en una "sociedad" en el sentido acotado de una totalidad social de base territorial, comprensiva de todas las dimensiones y esferas que son objeto de análisis de los sociólogos y, por tanto, "relativamente autosuficiente" (Pérez-Agote, 2012: 282).

Reflexiones de este tipo proliferan en las ciencias sociales desde finales de los años ochenta, justo cuando la expresión "nacionalismo metodológico" entra en el vocabulario usual del desconcierto sociológico (Beck, 1999; Wimmer y Glick Schiller, 2002). Algunos de estos cuestionamientos vienen motivados por la emergencia de la temática de las diásporas. Expresiones paradigmáticas del momento transnacional, las diásporas arriman a pensar en una tendencia a la dispersión de lo social que produce unas subjetividades diferenciadas respecto a la experiencia del sujeto centrado y legalmente acotado del Estado-nación.

\section{LO SOCIAL DISPERSO: EL REDESCUBRIMIENTO DE LAS DIÁSPORAS EN LAS CIENCIAS SOCIALES}

Hay cierto acuerdo entre los autores respecto a que los años noventa son los del redescubrimiento, y luego proliferación, de la temática de las diásporas en las ciencias sociales, singularmente las norteamericanas, que integran estas nuevas cuestiones en los estudios culturales, los estudios étnicos y en los area studies (Brah, 1996; Shuval, 2000; Butler, 2001; Braziel y Mannur, 2003). Al comenzar esta década, aparece una revista editada por Khachig Tölölyan, Diaspora. A Journal of Transnational Studies, la cual es explícita en su intención de publicar artículos escritos desde múltiples disciplinas, teorías e ideologías, dedicados a un único tema, el de las 
diásporas. Pero también anuncia de entrada que esta dedicación "pretende contribuir al estudio de todos los aspectos del transnacionalismo" (Tölölyan, 1991: 1) y que, a tal efecto, las diásporas constituyen "la comunidad ejemplar del momento transnacional" (Tölölyan, 1991: 4). El fenómeno de las diásporas es declarado en estrecha relación con el transnacionalismo, y más específicamente, como lo señala el editor en un extenso artículo de la misma publicación (Tölölyan, 1996: 5), con la llamada crisis del Estado-nación, en la medida que las diásporas exceden los patrones de territorialidad e identidad exclusiva que están implícitos en la institucionalidad política moderna. En este sentido, la categoría deviene "heurísticamente productiva" para cuestionar la soberanía de los Estados nacionales que, al son de los entusiastas discursos de la época sobre la globalización, parecía encaminarse inexorablemente a un "debilitamiento progresivo" (Schnapper, 1999: 229).

Pero, a pesar del prometedor descubrimiento de potencialidades críticas del concepto -o, precisamente por ello-, lo cierto es que no tardarán en llegar los llamados al orden semántico a través de diversos trabajos que proponen definiciones (Butler, 2001), sugieren criterios o requisitos (Safran, 1991; Tölölyan, 1996; Brubaker, 2005), describen concepciones (Vertovec y Cohen, 1999), trazan tipologías y casos (Shuval, 2000), o señalan rupturas históricas para diferenciar a las diásporas (Schnapper, 1999; Cohen, 1996). Brubaker (2005) se muestra especialmente atento a esta "diasporización" de significados del concepto, señalando incluso su extensión semántica no ya a grupos étnicos o de desplazamientos nacionales sino a casi cualquier población dispersa en el espacio: yankees, blancos, gays, comunidades digitales, etc. El problema que se presenta — señala también el autor-, es que el término culmina por no explicar nada, pierde su poder de discriminación y de establecer distinciones.

Ante tal situación, es necesario procurar cierto consenso en su definición (Butler, 2001: 190). Uno de los textos que sienta las bases para esta discusión es el de Safran (1991), ya que, quizá por su temprana aparición, es el que se encuentra reiteradamente citado, bien para adherir a su propuesta o bien para criticarla. En general, aun cuando puedan encontrarse matices entre las definiciones que proponen los diferentes autores, los elementos que permanecen constantes son, según Shuval (2000): una historia de la dispersión; una memoria o mito del lugar de procedencia, cierta sensación de no estar plenamente integrado en la sociedad de residencia, un deseo ambivalente de regreso y una identidad 
colectiva definida a partir de estas características. Lo que en todo caso sobresale, en opinión de la autora, son los componentes afectivoexpresivos de una identidad colectiva hecha de narrativas sobre un pasado común único que le otorga sentido al tiempo presente del grupo. Sobre estos elementos comunes, Shuval hace un llamado a diferenciar analíticamente entre tipos y subtipos de diásporas, para descubrir en éstas diferentes orientaciones, valores y actitudes. Por consiguiente, no cree la autora que sea útil una definición común y que, en cambio, una distinción tipológica permitiría reencauzar la cuestión hacia la producción de investigaciones empíricas ${ }^{3}$.

Pretendiendo evitar cierta tendencia a reificar la realidad social, Brubaker (2005) cree necesario asumir la diáspora como una "categoría de práctica", es decir como aquello que permite dar cuenta de movilizaciones, de lealtades, de generación de demandas sociales, de articulación de proyectos que pretenden cambiar el mundo y, luego, respetando esta prioridad, quizá sea fructífero hacer uso de la diáspora como una categoría de análisis ${ }^{4}$. De otra forma, nos previene Brubacker, se corre el riesgo de caer en una especie de "grupismo" que presupone la existencia de una colectividad etnodemográfica o etnocultural más o menos homogénea y unida. En cambio, si se habla de prácticas, proyectos o posiciones (stances) diaspóricas, se torna posible "estudiar empíricamente el grado y la forma de sostenimiento de un proyecto diaspórico entre los miembros de una determinada colectividad" (Brubaker, 2005: 13).

Abonando este afán de clarificación, la variable histórica es otra de las dimensiones que destacan en el tratamiento de las diásporas. Cohen (1996), por ejemplo, vuelve sobre el prístino pasado judío para subrayar la existencia de un tipo de diáspora traumática, víctima de desplazamientos, exilios o esclavitud. Para este caso, el autor subraya el trauma de la destrucción de Jerusalén como acontecimiento propiciador de la creación

\footnotetext{
${ }^{3}$ En este sentido, Brah también sugiere distinguir entre las diásporas según las condiciones económicas, sociales y políticas que le dan origen, es decir, en identificar el "régimen de poder" que está inscrito en la formación de cada diáspora, tanto en las circunstancias que propician la emigración del lugar de origen, como las que determinan el asentamiento. Así, según la autora, es fundamental que cada diáspora empírica sea analizada en sus circunstancias históricas pero también en el contexto de sus relaciones con otras diásporas (1996: 182-83).

${ }^{4}$ Podríamos así decir de la diáspora lo que Pérez-Agote dice de la nación: "no es un concepto científico"; su definición corresponde a los actores y, en todo caso, lo que les cabe a los científicos es evaluar su eficacia social, su capacidad para influir en el comportamiento de los actores sociales (Pérez-Agote, 1994: 27-31).
} 
de una memoria histórica negativa. Otros cuatro grupos compartirían con los judíos pasados de semejante valor: los africanos, por su la prolongada historia de esclavitud; los irlandeses, debido a la hambruna que se experimenta durante la segunda mitad del siglo XIX por efecto de la modernización de la agricultura y la reforma en el régimen de propiedad de la tierra; los armenios, como consecuencia del genocidio turco de principios del siglo $X X, y$ los palestinos, después de la creación del Estado de Israel en $1948^{5}$.

Siguiendo el hilo histórico de la modernidad, en la visión de Schnapper (1999) es la formación de las naciones modernas lo que le dio al término un significado esencialmente negativo. Desde el momento en que el vínculo político se territorializa, las diásporas, en cuanto constituyen lazos que exceden esa demarcación territorial, devienen "anómicas" (...), en tanto crean "espacios que escapan al control político" (1999: 229). Según Schnapper, será con el "debilitamiento del poder del Estado-nación y del patriotismo nacional" (1999: 227) que se revierta esta valoración y las diásporas adquieran un sentido positivo. Ya sea por una inminente transnacionalización de la vida social, que erosiona en parte la soberanía de los Estados ${ }^{6}$, o por la disociación entre la participación política, la actividad económica y la identidad nacional, que no quedan ya del todo integradas dentro de los confines territoriales, las diásporas representan, según la autora, un desafío al Zeitgeist de la modernidad. El movimiento de

\footnotetext{
${ }^{5}$ La lista podría sin duda ampliarse al tiempo de la redacción de este artículo. Estamos en presencia de la formación de una diáspora de memoria negativa a cuenta del éxodo sirio: una desvinculación violenta de una población con su territorio que se presenta como un atentado demográfico capaz de diseminar a más de 11 millones de personas fuera de las fronteras que contenían su existencia social.

${ }^{6}$ Schnapper sostiene que, en el caso europeo, este debilitamiento se manifiesta en cierta medida desde la firma del Acuerdo de Schengen, en 1985, mientras que en Estados Unidos - aun cuando podría considerarse que escapa a esta regla - se deja ver a partir de la creciente "etnización" de su política exterior (1999: 241). Son discutibles estas referencias que presenta Schnapper para dar cuenta del declive en la soberanía de los Estados nacionales. En el primer caso parece evidente que lo que se produce por la época es una flexibilización de los controles para dar salida a las poblaciones pero, en cambio, una mayor rigidez en la habilitación de los ingresos de la inmigración que haría dudar sobre la pérdida de capacidad soberana de los Estados. Para el caso de Estados Unidos, si bien algunos autores han subrayado esta tradición de etnización en el ejercicio de lobbying de ciertas diásporas hacia la política exterior norteamericana (Shain, 1994), es esta una cuestión que, como asimismo lo resalta Shain (1994), bien podría ser puesta en relación a su peculiar modelo pluralista de nación (Smith, 1991) y a esa especie de "religión civil" del nacionalismo estadounidense que es capaz de generar una dinámica centripeta y centrífuga a la vez, mitigando y acentuado la pertenencia étnica (Bellah, 1967).
} 
poblaciones había sido abordado por la Sociología en términos de emigración-inmigración. Ello acarreaba concepciones asimilacionistas que veían en las etnicidades e identidades resultantes de los procesos migratorios un "residuo simbólico" transitorio en el paso hacia la asimilación $y$, por lo tanto, estas identidades eran interpretadas como meramente "expresivas" y políticamente "marginales" (Cf. Gans, 1979). Lo que el nuevo momento histórico viene a señalar es el peso específico de las identidades y lo subjetivo en la conformación de la realidad social. Un redescubrimiento que se inscribe en el llamado "el giro subjetivo" de las ciencias sociales: la "razón del sujeto", que en décadas pasadas era relegada al lugar residual de la "ideología" y la "falsa conciencia", cobra ahora toda su entidad (Sarlo, 2006: 22). Si las instituciones modernas habian marcado los términos del equilibrio para la estabilidad de la conciencia individual y la contención relativa de las representaciones colectivas, se observa ahora cierta incapacidad del Estado-nación para centralizar programas institucionales que eviten la dispersión de la pertenencia colectiva y, en cierta medida, el quiebre de la propias nociones de individuo y sociedad (Touraine, 2005; Dubet, 2006). Como singularmente lo expresa Yúdice: en períodos de crisis como los actuales, "se disgregan y transforman los géneros conductuales que mantenían el mundo social en su lugar" (2006: 108) ${ }^{7}$. Ante tales panoramas proliferan entonces metodologías de análisis que procuran sobreponerse al dato simplemente agregativo privilegiando la experiencia subjetiva como reveladora de un "nosotros descentrado". Así, en la consideración de Brah, incluso la autobiografía, en tanto que narrativa que condensa el yo y el nosotros, se vuelve una técnica idónea para las ciencias sociales puesto que ponen de manifiesto unas subjetividades forjadas en la experiencia del desplazamiento. "La narración individual es fundamentalmente significativa como una rememoración colectiva", dice Brah (1996: 10), y en la diáspora se vuelve analíticamente promisoria de las relaciones entre afecto, modalidades psíquicas, relaciones sociales y políticas (1996: 5).

\footnotetext{
${ }^{7}$ Según Yúdice, "en El Salvador, la guerra civil de los 80 y la globalización han trasladado la territorialidad de la nación al mundo imaginado de la diáspora. El concepto de diáspora, tal como se ha elaborado en los estudios afroamericanos, procura dar cuenta de la articulación de los pueblos afrodescendientes heterogéneos en una serie de prácticas que constituyen la base de una identidad coproducida a distancia, debido no sólo ni primordialmente a una matriz originaria común (la patria), sino a las diversas maneras de imaginarla" (Yúdice, 2006: 109).
} 
Ya "no hay garantías en la naturaleza" (Hall, 2003: 91) para referir las identidades; hay en cambio denuncias del "absolutismo étnico" de la modernidad (Gilroy, 1993) con sus correlaciones entre territorio, etnicidad y Estado-nación y la identidad diaspórica, con su carácter trans o aterritorial, más que "un prius, objeto, sustrato, o sustancia esencial", "es un locus social", un lugar social imaginado e imaginario, producto de diversas fuerzas sociales (Mendieta, 2003: 408) que dan cuenta de dislocaciones diversas ${ }^{8}$. Se abren en este panorama nuevos campos de visualización etnográfica (scapes) (Appadurai, 1991) sobre fenómenos que permanecían gnoseológicamente ocultos bajo los Estados nacionales y las ciencias sociales enfrentan denuncias de prolongadas prácticas de "nacionalismo metodológico" por el hecho de ver reducidos sus objetos de análisis a la propia delimitación territorial que aquellos realizan (Wimmer y Glick Schiller, 2002: 307).

En relación a la migración, los tiempos globales devienen transnacionales, revierten el canon de la noción de asimilación en la medida en que se entiende que es posible ser parte de dos sociedades al mismo tiempo y extraer de ello ventajas política y económicas (Portes y DeWind, 2004). La nación excede las fronteras del Estado. Y no sólo para los individuos que replican en sentidos afectivos y prácticos la doble inserción; también para los gobiernos de los paises de origen que, o bien alientan la emigración para interpelar luego a su población del exterior como diáspora e integrar el producto de su trabajo en el desarrollo de sus comunidades de origen, como es el caso de México con respecto a su población en Estados Unidos (Irazuzta, 2008); o bien lo hacen como una oportunidad para incrementar internacionalmente la legitimidad de naciones sin Estado, como lo ilustra la renombrada como "diáspora vasca de los cinco continentes" a partir de los años noventa (Irazuzta, 2001). Con la diáspora, el nacionalismo del transnacionalismo va más allá del Estado, se hace "de larga distancia" (Anderson, 1998) y crea y sostiene sentido y acción social más allá del territorio significado como nacional.

\footnotetext{
${ }^{8}$ En tanto protagonistas ejemplares de tales procesos, las diásporas tornan problemática la posición de sujeto nativo y la propia noción de indígena en su sentido más elemental, es decir, como aquello que "indica la génesis". Despojadas así las identidades diaspóricas de su constitución material, es posible hablar de un "espacio diaspórico" habitado no sólo por aquellos que han migrado y sus descendientes, sino también por aquellos que son construidos y representados como indígenas: "el espacio diaspórico es el lugar donde el nativo es tan diaspórico como éste es nativo" (Brah, 1996: 209).
} 
La transformación del mundo y de los saberes que lo interpretan sugieren la pregunta acerca de si no estaremos asistiendo a un cambio de época que nos sitúa en el gozne que cierra la era del Estado-nación y abre otra de diásporas (Brubacker, 2005: 7-8). Extendiendo la potencialidad explicativa del términos diáspora, la pregunta arrima a la respuesta sobre una diasporización de las relaciones sociales y las instituciones políticas, una suerte de pérdida de anclaje de lo social, como proponen muchas sociologías contemporáneas y como en parte se lo sugiere en este trabajo.

\section{LUGARES EN EL MUNDO. REFLEXIONES FINALES}

La dispersión es la condición social de la diáspora y ésta es una distribución aleatoria en el espacio de valores procedentes de una identidad central. La diáspora, al menos en su versión nacional, es la emanación de ese "centro simbólico" que gestiona, dispone y administra la sacralidad del orden social en las sociedades modernas, pluralistas, seculares y territoriales (Shils, 1975; Pérez-Agote, 1984). En el estado de plenitud de estas sociedades, en la era del Estado-nación del siglo XIX y XX, la diáspora es una anomalía; es una desviación con respecto al vínculo territorial que constituye y sostiene el sentido del individuo y su sociedad. En las sociedades contemporáneas, globales y transnacionales, la anomalía se estabiliza, la diáspora se vuelve una situación normal por copiosa y se reproduce en el contexto de la multiplicación de centros (Pérez-Agote, 2012: 276) y de una experiencia social igualmente múltiple. Dos aspectos hacen a lo social disperso:

1. Desde la perspectiva distante y algo fría de una sociología interesada por las estructuras y lo instrumental, la desterritorialización del vínculo social, que da forma a demografías descentradas y cartografías sociales dispersas. Con las diásporas y el transnacionalismo pareciera que la nación excede al Estado, prolongándose de manera espectral respecto a la base territorial que hace a su objetivación política en la modernidad. En lo social disperso de la realidad transnacional, el nacionalismo pierde el telos que dirige la voluntad política hacia la construcción de una sociedad a la medida del Estado-nación para asentarse como dato empírico de nuevas escalas o ensamblajes sociales.

2. Desde otro costado sociológico más cálido, el sentido se disgrega y adquiere protagonismo en formas múltiples y plurales. Hay un descentramiento del sujeto respecto a las instituciones que gestionaban la legitimidad en lo "social acotado" de la nación, a la vez 
que una sobreexposición de las subjetividades. La experiencia del sujeto es reveladora de un nosotros descentrado y tiende a redefinir las relaciones entre territorio, autoridad y derechos (Sassen, 2013) que acomodaban la autonomía del sujeto de la modernidad plena que imaginó la Sociología en la era del Estado-nación.

Si "la modernidad ha sido más bien una excepción histórica, un periodo limitado de tiempo en el que se da una condensación territorial política de la vida social" (Pérez-Agote, 2012: 287), también parece ser cierto que en la era del transnacionalismo esa modernidad deriva en consecuencias no intencionadas de hogar y lugares en el mundo. Quizá como una rémora de antiguos vínculos, como un espectro de viejos campanilismos, algunas relaciones sociales lógicamente anómalas en la era del Estado-nación, pasen -como nuevamente lo sugiere Alfonso- a tener sentido en sí mismas. Lo social disperso es el gobierno de la vida por el sentido cuando el territorio ha perdido centralidad en la gestión política de la vida social.

\section{BibliografíA}

Appadurai, A., 1991, "Global Ethnoscapes. Notes and Queries for a Transnational Anthropology", en R. Fox (Ed.), Recapturing Anthropology, School of American Research Press, Santa Fe, New Mexico, pp. 191-210.

Anderson, B., 1998, Espectre of Comparisons. Nationalism, Southeast Asia and the World, Verso, Londres.

Beck, U., 1999, ¿Qué es la globalización?, Paidós, Barcelona.

Bellah, R., 1967, "Civil religion in America”, en Dedalus, 96, 1, pp. 1-121.

Brah, A., 1996, Cartographies of Diaspora. Contesting Identities, Routledge, Londres.

Braziel, J., Mannur, A., (Eds.), 2003, Theorizing Diaspora, Blackwell, Londres.

Brubaker, R., 2005, "The 'diaspora' diaspora", en Ethnic and Racial Studies, 28, 1, pp. 1-19.

Butler, K., 2001, "Defining Diaspora, Redefining Discourse", en Diaspora. A journal of Transnational Studies, 10, 2, pp. 189-220.

Cohen, R., 1996, "Diasporas and the Nation-State: from Victims to Challengers", en International Affairs, 72, 3, pp. 506-520.

Dubet, F., 2006, El declive de la institución. Profesiones, sujetos e individuos en la modernidad, Gedisa, Barcelona. 
Gans, H., 1979, "Symbolic Ethnicity: the Future of Ethnic Groups and Cultures in America", en Ethnic and Racial Studies, 2, 1, pp. 2-20.

Gilroy, P., 1993, The Black Atlantic: Modernity and Double Consciousness, Verso, Londres.

Hall, S., 2003, “New Ethnicities”, en L. Martín Alcoff y E. Mendieta, (Eds.), Identities. Class, Gender and Nationality, Blackwell, Londres, pp. 90-95.

Irazuzta, I., 2001, Argentina, una construcción ritual, Servicio Editorial de la Universidad del País Vasco, Bilbao.

Irazuzta, I., 2008, “Gobiernos de enlace: la población y la política más allá del Estado", en Iconos, 35, pp. 129-140.

Mendieta, E., 2003, "Afterword. Identities: Postcolonial and Global", en L. Martín Alcoff y E. Mendieta, (Eds.), Identities. Class, Gender and Nationality, Blackwell, Londres, pp. 407-416.

Pérez-Agote, A., 1989a, "Hacia una concepción sociológica de la nación”, en A. Pérez-Agote (ed.), Sociología del nacionalismo, Servicio Editorial de la Universidad del País Vasco, Bilbao.

Pérez-Agote, A., 1989b, La sociedad y lo social. Ensayos de Sociología, Servicio Editorial de la Universidad del País Vasco, Bilbao.

Pérez-Agote, A., 1994, "16 tesis sobre la arbitrariedad de ser colectivo nacional", en Revista de Occidente, 161, pp. 23-44.

Pérez-Agote, A., 2008, Las raices sociales del nacionalismo vasco, CIS, Madrid.

Pérez-Agote, A., 2012, "Las formas elementales de la vida religiosa: descomposición contemporánea de las ecuaciones durkheimianas", en Politica y Sociedad, 49, 2, pp. 273-292.

Portes, A., DeWind, J., 2004, "A Cross Atlantic Dialog: Progress of Research and Theory in the Study of International Migration", en International Migration Review, 38, 3, pp. 828-851.

Safran, W., 1991, "Diasporas in Modern Societies: Myths of Homeland and Return", en Diaspora. A Journal of Transnational Studies, 1, 1, pp. 8399.

Sarlo, B., 2006, Tiempo pasado. Cultura de la memoria y giro subjetivo. Una discusión, Siglo XXI, Buenos Aires.

Sassen, S., 2013, Territorio, autoridad y derechos. De los ensamblajes medievales a los ensamblajes globales, Katz, Buenos Aires. 
Schnapper, D., 1999, "From the Nation-State to the Transnational World: On the meaning and Usefulness of Diaspora", en Diaspora. A Journal of Transnational Studies, 8, 3, pp. 225-255.

Smith, A., 1991, "Tres conceptos de nación", en Revista de Occidente, 61, pp. 7-22.

Shain, Y., 1994, "Marketing the Democratic Creed Abroad: US Diasporic Politics in the Era of Multiculturalism", en Diaspora. A Journal of Transnational Studies, 3, 1, pp. 85-111.

Shuval, J., 2000, "Diaspora Migration: Definitional Ambiguities and Theoretical Paradigm", en International migration, Vol. 38, No 5, pp. 41-56.

Tölölyan, K., 1991, "In This Issue", en Diaspora: A Journal of Transnational Studies, Vol. 1, 1.

Tölölyan, K., 1996, "Rethinking Diaspora(s): Stateless Power in the Transnational Moment", en Diaspora. A Journal of Transnational Studies, 5, 1, pp. 3-35.

Touraine, A., 2005, Un nuevo paradigma. Para comprender el mundo de hoy, Paidós, Barcelona.

Vertovec, S., Cohen, R., 1999, "Introduction", en S. Vertovec y R. Cohen (Eds.), Migration, Diasporas and Transnationalism, Edward Elgar, Massachusetts, pp. xiii-xxviii.

Wimmer, A., Glick Schiller, N., 2002, "Methodological Nationalism and Beyond: Nation-State Building, Migration and the Social Sciences", en Global Networks, 2, 4, pp. 301-334.

Yúdice, G., 2006, “¿Una o varias identidades? Cultura, globalización y migraciones", en Nueva sociedad, 201, enero-febrero, pp. 106-116. 